OPEN ACCESS

Edited by:

Giovanni Sarnelli,

University of Naples Federico II, Italy

Reviewed by:

Dan-Lucian Dumitrașcu,

Iuliu Haţieganu University of Medicine

and Pharmacy, Romania

Maria Cecilia Giron,

University of Padua, Italy

*Correspondence:

Chin-Hsiao Tseng

ccktsh@ms6.hinet.net

Specialty section:

This article was submitted to Gastrointestinal and Hepatic Pharmacology,

a section of the journal

Frontiers in Pharmacology

Received: 01 July 2020 Accepted: 21 December 2020

Published: 16 February 2021

Citation:

Tseng C-H (2021) Chronic Metformin Therapy is Associated with a Lower Risk of Hemorrhoid in Patients with Type 2 Diabetes Mellitus. Front. Pharmacol. 11:578831. doi: 10.3389/fphar.2020.578831

\section{Chronic Metformin Therapy is Associated with a Lower Risk of Hemorrhoid in Patients with Type 2 Diabetes Mellitus}

\author{
Chin-Hsiao Tseng ${ }^{1,2,3 *}$ \\ ${ }^{1}$ Department of Internal Medicine, National Taiwan University College of Medicine, Taipei, Taiwan, ${ }^{2}$ Division of Endocrinology and \\ Metabolism, Department of Internal Medicine, National Taiwan University Hospital, Taipei, Taiwan, ${ }^{3}$ Division of Environmental \\ Health and Occupational Medicine of the National Health Research Institutes, Zhunan, Taiwan
}

Background: Metformin has anti-inflammatory property and reduces the risk of varicose vein in our previous study.

Aim: To investigate the risk of hemorrhoid, another common disease involving the hemorrhoidal venous plexus, in ever vs. never users of metformin in patients with type 2 diabetes mellitus.

Methods: This is a population-based retrospective cohort study. Patients with new-onset type 2 diabetes mellitus during 1999-2005 were enrolled from Taiwan's National Health Insurance. All patients who were alive on January 1, 2006 were followed up until December 31, 2011. Analyses were conducted in both an unmatched cohort of 152,347 ever users and 19,523 never users and in 19,498 propensity score (PS)-matched pairs of ever and never users. Traditional Cox regression and Cox regression incorporated with the inverse probability of treatment weighting (IPTW) using the PS were used to estimate hazard ratios.

Results: New-onset hemorrhoid was diagnosed in 8,211 ever users and 2025 never users in the unmatched cohort and in 1,089 ever users and 2022 never users in the matched cohort. The hazard ratio for ever vs. never users derived from the traditional Cox regression was 0.464 (95\% confidence interval: 0.440-0.488) in the unmatched cohort; and was $0.488(0.453-0.525)$ in the matched cohort. In the IPTW models, the hazard ratio was $0.464(0.442-0.487)$ in the unmatched cohort and was $0.492(0.457-0.530)$ in the matched cohort. A dose-response pattern was observed while comparing the tertiles of cumulative duration, cumulative dose and defined daily dose of metformin therapy to never users in all analyses. A risk reduction of approximately 40-50\% was consistently observed in various sensitivity analyses.

Conclusion: Chronic therapy with metformin in patients with type 2 diabetes mellitus is associated with a lower risk of hemorrhoid.

Keywords: diabetes mellitus, hemorrhoid, metformin, National Health Insurance, pharmacoepidemiology, Taiwan 


\section{INTRODUCTION}

Hemorrhoid is a very common disease that affects the anorectal area resulting in distal displacement of the anal cushions. Clinical presentations include vascular congestion, inflammation, itching, soiling, pain, bleeding and prolapse. The precise cause remains unknown but conditions that increase intra-abdominal pressure may increase the pressure in the hemorrhoidal venous plexus and precipitate its development. These include straining during constipation, chronic diarrhea, irritable bowel syndrome, pregnancy, delivery, obesity, lack of exercise, low-fiber diets, cigarette smoking, anal intercourse, long-time standing, cirrhosis with ascites, pelvic floor dysfunction and chronic cough (Johannsson et al., 2005; Helvaci et al., 2009; Mott et al., 2018; Yetkin and Ozturk, 2018; Ekici et al., 2019; Nagaraj et al., 2019). Hemorrhoid is age-related with peak prevalence at the age of 45-65 years. Its prevalence was 39\% in a routine colorectal cancer screening conducted in Vienna, Austria and among them 55\% were asymptomatic (Riss et al., 2012). Medical management, dietary modification and behavioral therapies are initial treatment, but surgical interventions may be necessary in some patients (Mott et al., 2018).

Hemorrhoid and varicose veins share common pathophysiology and may have similar risk factors (Mott et al., 2018; Yetkin and Ozturk, 2018; Ekici et al., 2019). Metformin is now recommended by major treatment guidelines as the first-line therapy for patients with type 2 diabetes mellitus (American Diabetes Association, 2017; Salvatore et al., 2020) because of its multiple benefits beyond glycemic control, including immune modification, anti-inflammation, anti-atherosclerosis, anticancer and anti-aging (Wang et al., 2017). Our recent study suggested that use of metformin in patients with type 2 diabetes mellitus is also associated with a lower risk of varicose veins (Tseng, 2020). To our knowledge, there has been no previous study investigating whether metformin use might reduce the risk of hemorrhoid. Because it is reasonable to speculate that the beneficial effect of metformin on varicose veins might also be applied to hemorrhoid, the purpose of the present study was to evaluate whether metformin use in patients with type 2 diabetes mellitus could be associated with a lower risk of hemorrhoid, by using a nationwide reimbursement database of the Taiwan's National Health Insurance (NHI) and comparing the risk of hemorrhoid between ever users and never users of metformin, in both an unmatched cohort and a cohort of 1:1 matched pairs of ever and never users who were well balanced in characteristics based on propensity score (PS).

\section{MATERIALS AND METHODS}

Taiwan has implemented a universal and compulsory healthcare system, the NHI, since March 1995. More than 99\% of Taiwan's population is covered by the NHI. The Bureau of the NHI has contracts with all hospitals and $93 \%$ of all medical settings, and keeps all computer records of disease diagnoses, medication prescriptions and clinical procedures submitted for reimbursement purpose. After ethics review and approval by the Research Ethics Committee of the National Health Research Institutes, the reimbursement database can be used for academic research. Informed consent from the patients was not required according to the local regulations because all personal information has been de-identified for the protection of privacy. The present study was granted an approval number of 99274.

Disease diagnoses during the study period were coded by the International Classification of Diseases, Ninth Revision, Clinical Modification (ICD-9-CM). Diabetes was coded 250.XX and hemorrhoid was coded 455 .

The database has been described in more detail in a previously published paper (Tseng, 2017). The procedures used to enroll an unmatched original cohort and a cohort of 1:1 PS-matched pairs of ever and never users of metformin derived from the original cohort are shown in Figure 1. At first, we identified 423,949 patients with a new diagnosis of diabetes mellitus during 1999-2005 in the outpatient clinics and having been prescribed antidiabetic drugs for 2 or more times in the database. The following ineligible patients were then excluded: 1) ever users of metformin who had been prescribed other antidiabetic drugs before the initiation of metformin $(n=183,837) ; 2)$ patients with a diagnosis of type 1 diabetes mellitus $(n=2,062), 3)$ patients with missing data $(n=420), 4)$ patients with a diagnosis of hemorrhoid before entry or within 6 months of the diagnosis of diabetes mellitus ( $n=29,235), 5)$ patients with a diagnosis of any cancer before entry or within 6 months of the diagnosis of diabetes mellitus $(n=21,206), 6)$ patients who had been followed up for $<180$ days $(n=15,319)$. As a result, 152,347 ever users of metformin and 19,523 never users of metformin were identified and they were considered as the unmatched original cohort. All characteristics shown in Table 1 plus the date of entry were then used to create the PS by logistic regression. A cohort of 19,498 PS-matched pairs of ever users and never users of metformin (the matched cohort) was then created from the unmatched cohort by using the Greedy $8 \rightarrow 1$ digit match algorithm proposed by Parsons (Parsons, 2020). The following analyses were conducted in both the unmatched cohort and the matched cohort to examine the consistency of the findings.

Potential confounders were categorized into the following subgroups: demographic data, major comorbidities, diabetesrelated complications, antidiabetic drugs, commonly encountered comorbidities and potential risk factors and commonly used medications in patients with diabetes mellitus. Demographic data included age, sex, occupation and living region. Major comorbidities included in the analyses were hypertension (401-405), dyslipidemia (272.0-272.4) and obesity (278). Diabetes-related complications included nephropathy (580-589), eye diseases (250.5: diabetes with ophthalmic manifestations, 362.0: diabetic retinopathy, 369: blindness and low vision, 366.41: diabetic cataract, and 365.44: glaucoma associated with systemic syndromes), diabetic polyneuropathy (357.2 and 250.6), stroke (430-438), ischemic heart disease (410-414) and peripheral arterial disease (250.7, 785.4, 443.81 and 440-448). Antidiabetic drugs were categorized as insulin, sulfonylurea, meglitinide, acarbose, rosiglitazone and pioglitazone. Commonly encountered comorbidities and 


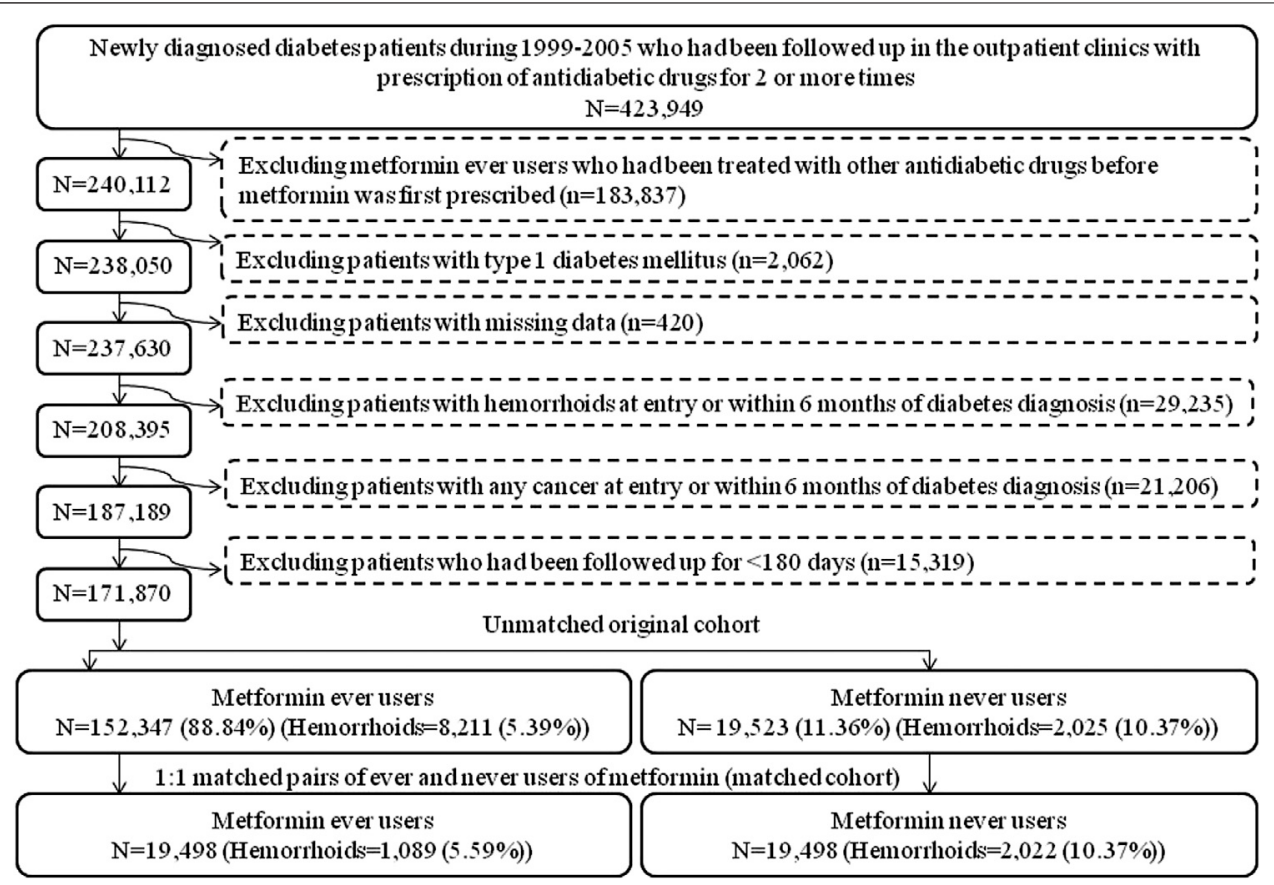

FIGURE 1 | Flowchart showing the procedures in creating an unmatched cohort and a cohort of 1:1 matched-pairs (based on propensity score) of metformin ever users and never users from the reimbursement database of the Taiwan's National Health Insurance.

potential risk factors of hemorrhoid included chronic obstructive pulmonary disease (a surrogate for smoking, 490-496), tobacco abuse (305.1, 649.0 and 989.84), alcohol-related diagnoses (291, $303,535.3,571.0-571.3$ and 980.0$)$, cancer (140-208), heart failure $(398.91,402.11,402.91,404.11,404.13,404.91,404.93$ and 428), Parkinson's disease (332), dementia (abridged codes of A210 or A222, or ICD-9-CM codes of 290.0, 290.1, 290.2, 290.4, 294.1, 331.0-331.2 and 331.7-331.9), head injury (959.01) and valvular heart disease (394-396, 424 and 746). Medications commonly used by patients with diabetes mellitus included angiotensin-converting enzyme inhibitors/angiotensin receptor blockers, calcium channel blockers, statins, fibrates and aspirin.

The living region and occupation were described in detail elsewhere (Tseng, 2012). In brief, the living region was classified as Taipei, Northern, Central, Southern, and Kao-Ping/Eastern. Occupation was classified as class I (civil servants, teachers, employees of governmental or private businesses, professionals and technicians), class II (people without a specific employer, selfemployed people or seamen), class III (farmers or fishermen) and class IV (low-income families supported by social welfare, or veterans).

Standardized difference for each of the above potential confounders was calculated as a test of balance diagnostic according to Austin and Stuart (2015). A cutoff value of $>10 \%$ was used as an indication of potential confounding from the variable.

Cumulative duration of metformin therapy in months, cumulative dose of metformin therapy in grams and units of defined daily dose (DDD) of metformin use per day [1 unit of DDD for metformin $=2 \mathrm{~g}$ (Chang et al., 2018)] were calculated and their tertiles were used for dose-response analyses. Incidence density of hemorrhoid was calculated for never users of metformin, ever users of metformin, the tertiles of cumulative duration of metformin therapy, the tertiles of cumulative dose of metformin therapy and the tertiles of units of DDD of metformin use per day. Start of follow-up was set on January 1, 2006. The numerator of the incidence was the case number of newly identified hemorrhoid during follow-up. The denominator (expressed in person-years) was the follow-up duration between the start of follow-up and the time of a new diagnosis of hemorrhoid, or the date of death or the date of the last reimbursement record, whichever occurred first up to December 31, 2011.

Kaplan-Meier curves of hemorrhoid-free probability were plotted for never users and ever users of metformin, for never users and tertiles of cumulative duration of metformin therapy, for never users and tertiles of cumulative dose of metformin therapy and for never users and tertiles of units of DDD per day. The significance in different subgroups of metformin exposure was tested by logrank test.

The subgroup of never users of metformin was used as the referent group in the estimation of hazard ratios and their 95\% confidence intervals for hemorrhoid for ever users and for each tertile of cumulative duration, cumulative dose and units of DDD. Both traditional Cox regression and Cox regression incorporated with the inverse probability of treatment weighting (IPTW) using the PS were used to examine the consistency of the findings. The IPTW method was proposed by Austin to reduce the potential confounding from the differences in characteristics (Austin, 2013). 
TABLE 1 | Characteristics in never and ever users of metformin in the unmatched cohort and the matched cohort.

\begin{tabular}{|c|c|c|c|c|c|c|c|c|c|c|}
\hline \multirow[t]{3}{*}{ Variable } & \multicolumn{5}{|c|}{ Unmatched cohort } & \multicolumn{5}{|c|}{ Matched cohort } \\
\hline & \multicolumn{2}{|c|}{$\begin{array}{c}\text { Never users } \\
(n=19,523)\end{array}$} & \multicolumn{2}{|c|}{$\begin{array}{c}\text { Ever users } \\
(n=152,347)\end{array}$} & \multirow[t]{2}{*}{ SD } & \multicolumn{2}{|c|}{$\begin{array}{c}\text { Never users } \\
(n=19,498)\end{array}$} & \multicolumn{2}{|c|}{$\begin{array}{l}\text { Ever users } \\
(n=19,498)\end{array}$} & \multirow[t]{2}{*}{ SD } \\
\hline & $n$ & $\%$ & $n$ & $\%$ & & $n$ & $\%$ & $n$ & $\%$ & \\
\hline \multicolumn{11}{|l|}{ Demographic data } \\
\hline $\mathrm{Age}^{\mathrm{a}}$ (years) & 68.32 & 13.34 & 64.11 & 11.94 & -39.37 & 68.30 & 13.33 & 68.09 & 12.29 & -1.09 \\
\hline Sex (men) & 10,548 & 54.03 & 79,205 & 51.99 & -4.11 & 10,537 & 54.04 & 10,430 & 53.49 & -1.28 \\
\hline \multicolumn{11}{|l|}{ Occupation } \\
\hline 1 & 7,039 & 36.05 & 56,816 & 37.29 & & 7,032 & 36.07 & 7,010 & 35.95 & \\
\hline$\|$ & 3,249 & 16.64 & 32,363 & 21.24 & 13.52 & 3,246 & 16.65 & 3,273 & 16.79 & 0.25 \\
\hline III & 4,814 & 24.66 & 35,482 & 23.29 & -3.51 & 4,809 & 24.66 & 4,885 & 25.05 & 1.21 \\
\hline IV & 4,421 & 22.65 & 27,686 & 18.17 & -13.11 & 4,411 & 22.62 & 4,330 & 22.21 & -0.89 \\
\hline \multicolumn{11}{|l|}{ Living region } \\
\hline Taipei & 6,518 & 33.39 & 47,352 & 31.08 & & 6,508 & 33.38 & 6,404 & 32.84 & \\
\hline Northern & 2,061 & 10.56 & 17,604 & 11.56 & 3.38 & 2,061 & 10.57 & 2,057 & 10.55 & -0.04 \\
\hline Central & 3,359 & 17.21 & 27,594 & 18.11 & 2.21 & 3,353 & 17.20 & 3,391 & 17.39 & 0.49 \\
\hline Southern & 3,438 & 17.61 & 26,418 & 17.34 & -0.56 & 3,435 & 17.62 & 3,447 & 17.68 & 0.40 \\
\hline Kao-Ping and Eastern & 4,147 & 21.24 & 33,379 & 21.91 & 2.61 & 4,141 & 21.24 & 4,199 & 21.54 & 0.86 \\
\hline \multicolumn{11}{|l|}{ Major comorbidities } \\
\hline Hypertension & 16,513 & 84.58 & 126,762 & 83.21 & -5.14 & 16,489 & 84.57 & 16,474 & 84.49 & -0.03 \\
\hline Dyslipidemia & 13,461 & 68.95 & 123,784 & 81.25 & 33.25 & 13,452 & 68.99 & 13,535 & 69.42 & 0.92 \\
\hline Obesity & 452 & 2.32 & 6,212 & 4.08 & 10.69 & 452 & 2.32 & 479 & 2.46 & 0.90 \\
\hline \multicolumn{11}{|l|}{ Diabetes-related complications } \\
\hline Nephropathy & 7,173 & 36.74 & 43,358 & 28.46 & -22.29 & 7,153 & 36.69 & 7,083 & 36.33 & -1.15 \\
\hline Eye diseases & 3,284 & 16.82 & 48,021 & 31.52 & 36.51 & 3,283 & 16.84 & 3,140 & 16.10 & -2.60 \\
\hline Diabetic polyneuropathy & 3,302 & 16.91 & 44,227 & 29.03 & 30.82 & 3,302 & 16.94 & 3,272 & 16.78 & -0.78 \\
\hline Stroke & 7,710 & 39.49 & 49,996 & 32.82 & -18.19 & 7,689 & 39.43 & 7,562 & 38.78 & -1.22 \\
\hline Ischemic heart disease & 10,191 & 52.20 & 72,530 & 47.61 & -11.73 & 10,175 & 52.18 & 10,001 & 51.29 & -1.62 \\
\hline Peripheral arterial disease & 4,864 & 24.91 & 40,714 & 26.72 & 2.95 & 4,854 & 24.89 & 4,733 & 24.27 & -1.59 \\
\hline \multicolumn{11}{|l|}{ Antidiabetic drugs } \\
\hline Insulin & 1,658 & 8.49 & 3,487 & 2.29 & -34.88 & 1,641 & 8.42 & 1,489 & 7.64 & -5.15 \\
\hline Sulfonylurea & 14,157 & 72.51 & 109,708 & 72.01 & 8.27 & 14,155 & 72.60 & 14,649 & 75.13 & 4.77 \\
\hline Meglitinide & 1,695 & 8.68 & 6,113 & 4.01 & -21.47 & 1,689 & 8.66 & 1,681 & 8.62 & -0.56 \\
\hline Acarbose & 2,138 & 10.95 & 8,189 & 5.38 & -19.59 & 2,131 & 10.93 & 2,301 & 11.80 & 0.79 \\
\hline Rosiglitazone & 558 & 2.86 & 7,270 & 4.77 & 11.29 & 558 & 2.86 & 613 & 3.14 & 0.72 \\
\hline Pioglitazone & 444 & 2.27 & 3,866 & 2.54 & 3.24 & 443 & 2.27 & 473 & 2.43 & 0.17 \\
\hline \multicolumn{11}{|l|}{ Commonly encountered comorbidities and potential risk factors } \\
\hline Chronic obstructive pulmonary disease & 10,476 & 53.66 & 76,095 & 49.95 & -10.34 & 10,454 & 53.62 & 10,478 & 53.74 & 0.45 \\
\hline Tobacco abuse & 440 & 2.25 & 5,437 & 3.57 & 8.61 & 440 & 2.26 & 394 & 2.02 & -1.70 \\
\hline Alcohol-related diagnoses & 1,188 & 6.09 & 9,438 & 6.20 & 0.39 & 1,188 & 6.09 & 1,220 & 6.26 & 0.55 \\
\hline Cancer & 1,822 & 9.33 & 11,224 & 7.37 & -7.67 & 1,817 & 9.32 & 1,872 & 9.60 & 0.94 \\
\hline Heart failure & 5,462 & 27.98 & 30,250 & 19.86 & -24.05 & 5,449 & 27.95 & 5,267 & 27.01 & -2.03 \\
\hline Parkinson's Disease & 1,120 & 5.74 & 5,625 & 3.69 & -12.10 & 1,112 & 5.70 & 1,047 & 5.37 & -1.30 \\
\hline Dementia & 2,196 & 11.25 & 10,980 & 7.21 & -19.61 & 2,508 & 12.86 & 2,364 & 12.12 & -2.05 \\
\hline Head injury & 730 & 3.74 & 6,034 & 3.96 & 1.18 & 729 & 3.74 & 691 & 3.54 & -1.09 \\
\hline Valvular heart disease & 2,791 & 14.30 & 15,552 & 10.21 & -16.13 & 2,785 & 14.28 & 2,707 & 13.88 & -1.05 \\
\hline \multicolumn{11}{|l|}{ Commonly used medications in diabetes patients } \\
\hline Angiotensin converting enzyme inhibitors/angiotensin receptor blockers & 14,238 & 72.93 & 113,939 & 74.79 & 3.61 & 14,216 & 72.91 & 14,178 & 72.72 & -0.38 \\
\hline Calcium channel blockers & 13,118 & 67.19 & 94,952 & 62.33 & -12.16 & 13,099 & 67.18 & 13,036 & 66.86 & -0.43 \\
\hline Statins & 10,002 & 51.23 & 98,120 & 64.41 & 30.28 & 9,999 & 51.28 & 9,958 & 51.07 & -0.66 \\
\hline Fibrates & 6,239 & 31.96 & 63,635 & 41.77 & 23.11 & 6,237 & 31.99 & 6,161 & 31.60 & -0.84 \\
\hline Aspirin & 11,985 & 61.39 & 96,528 & 63.36 & 2.65 & 11,966 & 61.37 & 12,019 & 61.64 & 0.70 \\
\hline
\end{tabular}

${ }^{a}$ Age is expressed as mean and standard deviation.

Refer to "Materials and Methods" for the classification of occupation.

$S D$ : standardized difference.

Sensitivity analyses were conducted by estimating the overall hazard ratios for ever users vs. never users in more homogeneous subgroups of patients. First, patients with irregular refill of metformin were excluded. This was done by excluding patients who received two consecutive prescriptions of metformin spanning a period of $>4$ months (Model I). Because the NHI allows drug prescription for chronic diseases for not more than 3 months at each time, these patients represented those who have delayed refill of metformin for more than one month after a previous prescription for 3 months. Second, patients who happened to be treated with incretin-based therapies, either with a dipeptidyl peptidase 4 inhibitor or a 

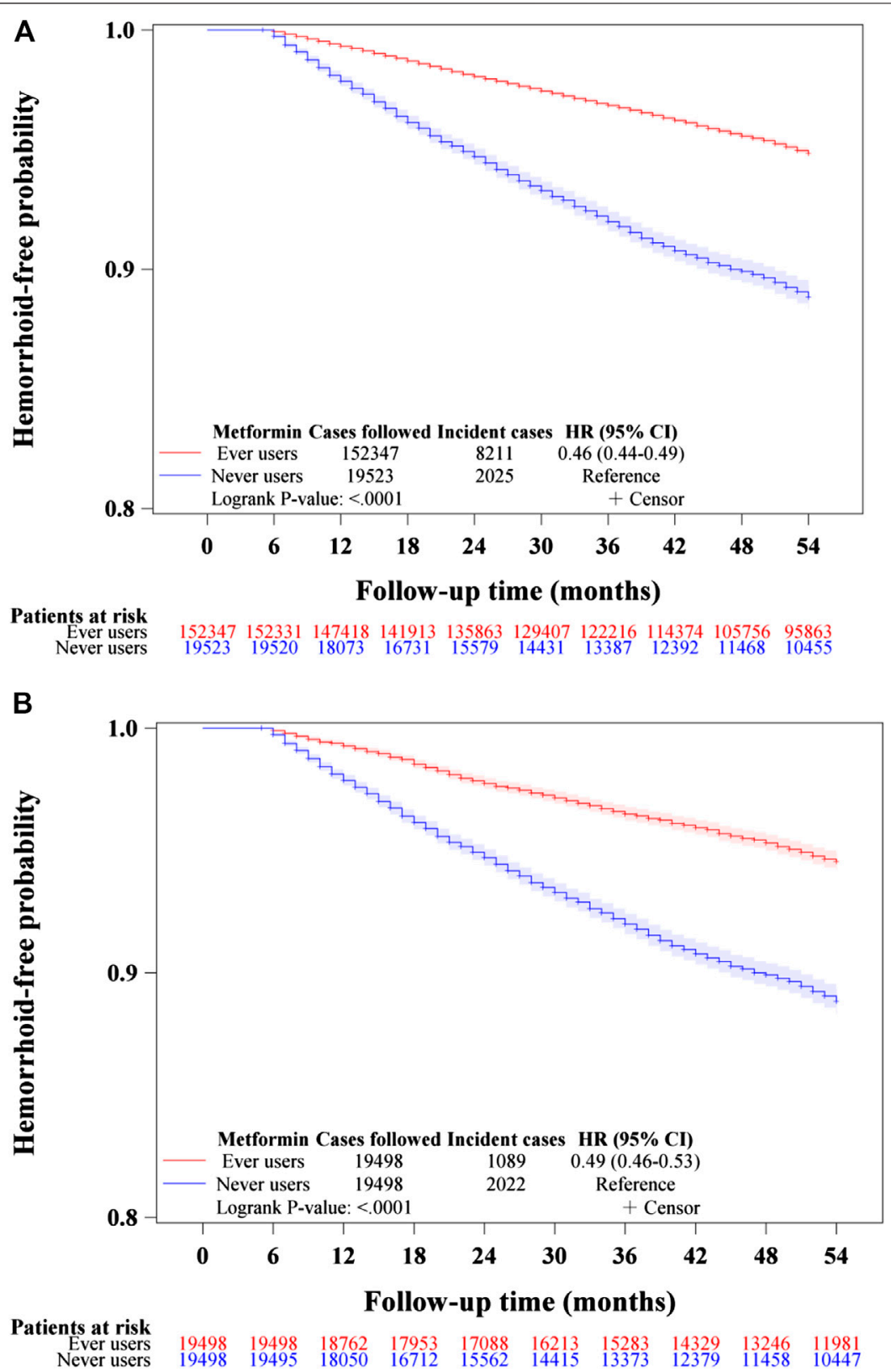

FIGURE 2 | Kaplan-Meier curves comparing hemorrhoid-free probability in never users and ever users of metformin in the unmatched cohort (A) and the matched cohort (B). HR: hazard ratio, Cl: confidence interval.

glucagon-like peptide 1 receptor agonist, during the follow-up period were excluded (Model II). In Taiwan, the first incretinbased therapy was not reimbursed by the NHI until after 2009. The exclusion of these patients avoided the potential impact of incretin-based therapies during follow-up. Third, patients enrolled during two different periods of 1999-2002 (Model III) and 2003-2005 (Model IV) were analyzed separately. Because more and more antidiabetic drugs have been introduced into clinical use and the guidelines for the use of antidiabetic drugs have evolved over the last 2 decades, these sensitivity analyses examined whether the results could be influenced by these changes. Fourth, to reduce the potential risk of misdiagnosis and misclassification of hemorrhoid at the outpatient clinics, analysis was performed by re-defining the outcome of hemorrhoid by using a more stringent criteria, i.e., as a primary diagnosis at hospitalization (Model V). These hospitalized patients might represent those who had more severe clinical manifestations of hemorrhoid and surgical intervention or more intensive medical care was required. Fifth, subgroup analyses were conducted with regards to the use of aspirin (Model VI: patients receiving aspirin; Model VII: patients not receiving aspirin) and calcium channel blockers (Model VIII: patients 


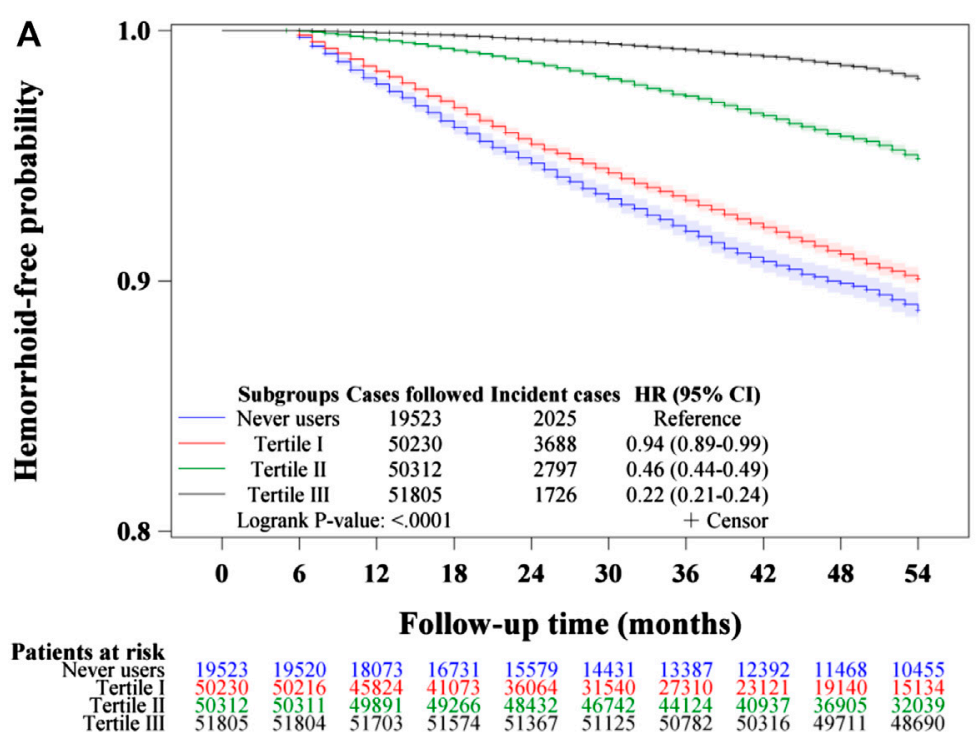

B

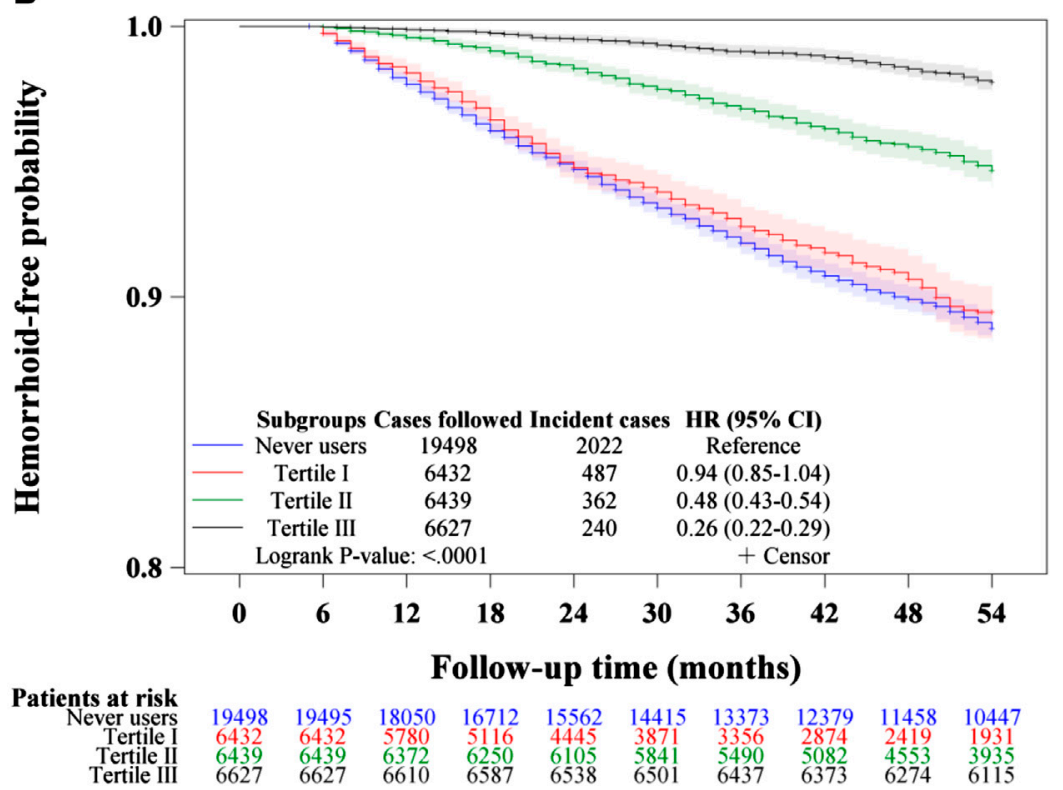

FIGURE 3 | Kaplan-Meier curves comparing hemorrhoid-free probability among never users and tertiles of cumulative duration of metformin therapy in the unmatched cohort (A) and the matched cohort (B), respectively. HR: hazard ratio, Cl: confidence interval.

receiving calcium channel blockers; Model IX: patients not receiving calcium channel blockers) because these medications may potentially cause bias relating to disease diagnosis. Aspirin can increase the risk of hemorrhoidal bleeding (Davis et al., 2018) but on the other hand it may also be used for hemorrhoidal pain relief (Sun and Migaly, 2016). Calcium channel blockers can reduce resting anal pressure and have been used for the treatment of hemorrhoid (Lohsiriwat, 2012) and anal fissure (Sahebally et al., 2017).

Analyses were conducted using SAS statistical software, version 9.4 (SAS Institute, Cary, NC). $p<0.05$ was considered statistically significant.

\section{RESULTS}

The characteristics in never users and ever users of metformin in the unmatched cohort and the matched cohort, respectively, are shown in Table 1. Many of the covariates were not balanced between never and ever users of metformin as indicated by a standardized difference $>10 \%$ in the unmatched cohort. However, all covariates were well balanced between the two groups in the matched cohort because none of them had a value of standardized difference $>10 \%$.

The Kaplan-Meier curves comparing hemorrhoid-free probability with regards to metformin exposure are shown in 

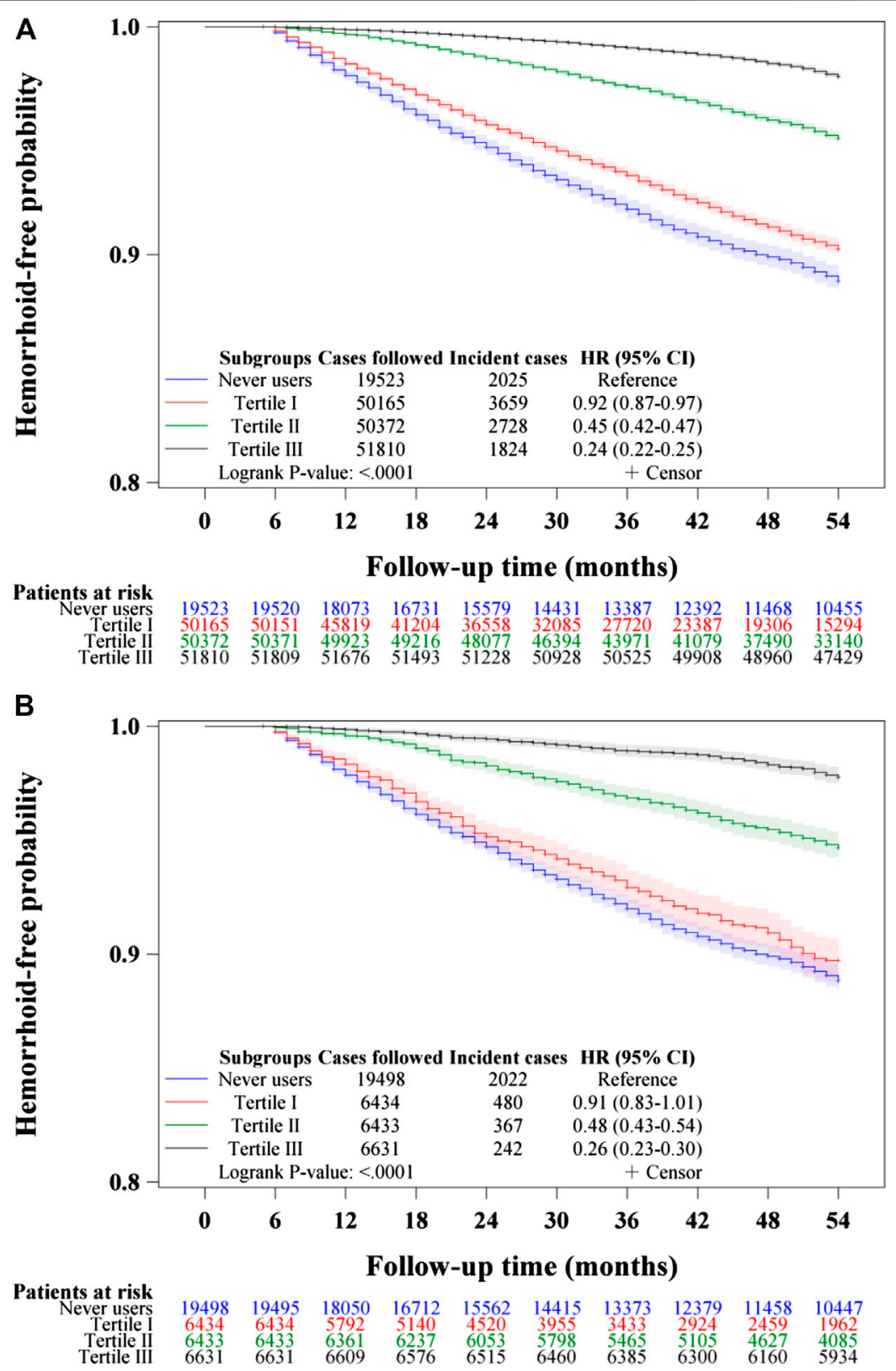

FIGURE 4 | Kaplan-Meier curves comparing hemorrhoid-free probability among never users and tertiles of cumulative dose of metformin therapy in the unmatched cohort (A) and the matched cohort $\mathbf{( B )}$, respectively.

Figure 2. Figure 2A shows the curves for never users and ever users in the unmatched cohort and Figure $\mathbf{2 B}$ shows the respective curves in the matched cohort. The $p$-values of the logrank test were $<0.0001$ in both the unmatched cohort and the matched cohort.

Figures 3-5 show the Kaplan-Meier curves comparing hemorrhoid-free probability in never users of metformin and in ever users of metformin categorized according to the tertiles of cumulative duration of metformin therapy (Figure 3), the tertiles of cumulative dose of metformin therapy (Figure 4) and the tertiles of units of DDD per day (Figure 5), respectively. Figures
3A, 4A, and 5A show the curves in the unmatched cohort; and Figures $\mathbf{3 B}, \mathbf{4 B}$, and $\mathbf{5 B}$ show the respective curves in the matched cohort. The logrank test $(p<0.0001)$ supported a significant difference in a dose-response pattern among the various subgroups of metformin exposure in all three parameters.

Table 2 shows the incidence of hemorrhoid and the hazard ratios by metformin exposure in the unmatched cohort and the matched cohort, respectively. A significantly lower risk in ever users could be demonstrated by the overall hazard ratios in both the traditional Cox regression and the Cox regression incorporated with IPTW in either the unmatched cohort or 

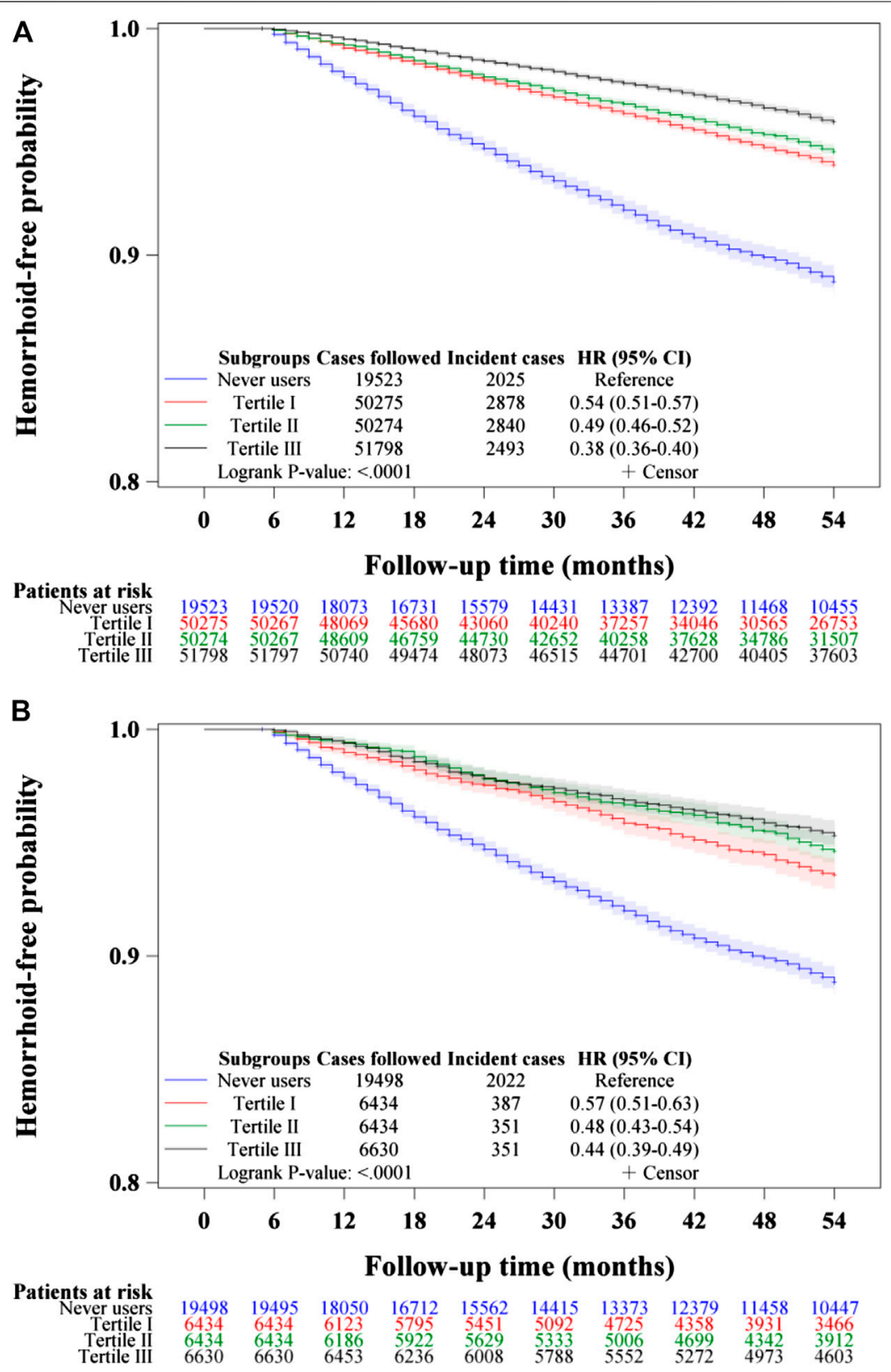

FIGURE 5 | Kaplan-Meier curves comparing hemorrhoid-free probability among never users and tertiles of units of defined daily dose of metformin in the unmatched cohort (A) and the matched cohort (B), respectively.

the matched cohort. A dose-response relationship could be seen in the tertile analyses in all models. Significant $p$-values were noted for metformin use for more than approximately 2 years in the cumulative duration analyses (in the second and third tertiles); for more than approximately 750 grams in the cumulative dose analyses (in the second and third tertiles); and for all tertiles in the units of DDD per day analyses. Analyses in the tertiles of units of DDD suggested that the protective effect could be seen across all tertiles with a trend of greater protection in higher daily dose. In the unmatched cohort, the mean (median) values of cumulative duration, cumulative dose and units of DDD of metformin therapy among ever users were 45.7 (40.6) years, $1,692.7(1,300.0)$ grams and $0.58(0.54)$ units of DDD, respectively. In the matched cohort, the respective values were 45.0 (40.1) years, $1,650.1(1,265.7)$ grams and $0.57(0.54)$ units of DDD.

The sensitivity analyses shown in Table $\mathbf{3}$ consistently supported a $40-50 \%$ lower risk of hemorrhoid associated with metformin use in models derived from the unmatched and matched cohorts, respectively; and in either the traditional Cox regression or the Cox regression incorporated with IPTW. The protective effect of metformin was independent of the use of 
TABLE 2 | Incidence rates of hemorrhoid and hazard ratios by metformin exposure.

\begin{tabular}{|c|c|c|c|c|c|c|c|c|c|c|}
\hline \multirow{2}{*}{$\begin{array}{l}\text { Model/Metformin } \\
\text { use }\end{array}$} & \multirow{2}{*}{$\begin{array}{l}\text { Incident } \\
\text { Case } \\
\text { number }\end{array}$} & \multirow{2}{*}{$\begin{array}{l}\text { Cases } \\
\text { Followed }\end{array}$} & \multirow[t]{2}{*}{ Person-years } & \multirow{2}{*}{$\begin{array}{c}\text { Incidence } \\
\text { rate } \\
\text { (per } \\
100,000 \\
\text { person-years) }\end{array}$} & \multicolumn{3}{|c|}{ Traditional Cox model } & \multicolumn{3}{|c|}{ IPTW model } \\
\hline & & & & & HR & $95 \% \mathrm{Cl}$ & p Value & HR & $95 \% \mathrm{Cl}$ & p Value \\
\hline \multicolumn{11}{|l|}{ Unmatched cohort } \\
\hline Never users & 2,025 & 19,523 & $80,153.12$ & $2,526.41$ & 1.000 & & & 1.000 & & \\
\hline Ever users & 8,211 & 152,347 & $692,486.07$ & $1,185.73$ & 0.464 & $(0.440-0.488)$ & $<0.0001$ & 0.464 & $(0.442-0.487)$ & $<0.0001$ \\
\hline \multicolumn{11}{|c|}{ Tertiles of cumulative duration of metformin therapy (months) } \\
\hline Never users & 2,025 & 19,523 & $80,153.12$ & $2,526.41$ & 1.000 & & & 1.000 & & \\
\hline$<25.5$ & 3,688 & 50,230 & $165,167.79$ & $2,232.88$ & 1.038 & $(0.978-1.101)$ & 0.2170 & 0.874 & $(0.827-0.923)$ & $<0.0001$ \\
\hline $25.5-56.7$ & 2,797 & 50,312 & $236,249.97$ & $1,183.92$ & 0.477 & $(0.449-0.506)$ & $<0.0001$ & 0.459 & $(0.434-0.486)$ & $<0.0001$ \\
\hline$>56.7$ & 1,726 & 51,805 & $291,068.31$ & 592.99 & 0.217 & $(0.203-0.232)$ & $<0.0001$ & 0.218 & $(0.205-0.233)$ & $<0.0001$ \\
\hline \multicolumn{11}{|c|}{ Tertiles of cumulative dose of metformin therapy (grams) } \\
\hline Never users & 2,025 & 19,523 & $80,153.12$ & $2,526.41$ & 1.000 & & & 1.000 & & \\
\hline$<756$ & 3,659 & 50,165 & $166,490.45$ & $2,197.72$ & 1.019 & $(0.960-1.081)$ & 0.5398 & 0.864 & $(0.818-0.912)$ & $<0.0001$ \\
\hline 756-1960 & 2,728 & 50,372 & $238,241.26$ & $1,145.06$ & 0.455 & $(0.429-0.484)$ & $<0.0001$ & 0.444 & $(0.420-0.471)$ & $<0.0001$ \\
\hline$>1960$ & 1,824 & 51,810 & $287,754.36$ & 633.87 & 0.231 & $(0.216-0.246)$ & $<0.0001$ & 0.235 & $(0.221-0.251)$ & $<0.0001$ \\
\hline \multicolumn{11}{|c|}{ Tertiles of units of defined daily dose of metformin therapy per day } \\
\hline Never users & 2,025 & 19,523 & $80,153.12$ & $2,526.41$ & 1.000 & & & 1.000 & & \\
\hline$<0.49$ & 2,878 & 50,275 & $211,323.75$ & $1,361.89$ & 0.531 & $(0.500-0.564)$ & $<0.0001$ & 0.536 & $(0.507-0.568)$ & $<0.0001$ \\
\hline $0.49-0.65$ & 2,840 & 50,274 & $227,907.44$ & $1,246.12$ & 0.488 & $(0.459-0.518)$ & $<0.0001$ & 0.489 & $(0.462-0.517)$ & $<0.0001$ \\
\hline$>0.65$ & 2,493 & 51,798 & $253,254.89$ & 984.38 & 0.387 & $(0.364-0.411)$ & $<0.0001$ & 0.382 & $(0.360-0.405)$ & $<0.0001$ \\
\hline \multicolumn{11}{|l|}{ Matched cohort } \\
\hline Never users & 2,022 & 19,498 & $80,068.10$ & $2,525.35$ & 1.000 & & & 1.000 & & \\
\hline Ever users & 1,089 & 19,498 & $87,196.70$ & $1,248.90$ & 0.488 & $(0.453-0.525)$ & $<0.0001$ & 0.492 & $(0.457-0.530)$ & $<0.0001$ \\
\hline \multicolumn{11}{|c|}{ Tertiles of cumulative duration of metformin therapy (months) } \\
\hline Never users & 2,022 & 19,498 & $80,068.10$ & $2,525.35$ & 1.000 & & & 1.000 & & \\
\hline$<24.9$ & 487 & 6,432 & $20,745.05$ & $2,347.55$ & 0.985 & $(0.888-1.092)$ & 0.7740 & 0.915 & $(0.828-1.011)$ & 0.0799 \\
\hline $24.9-56.0$ & 362 & 6,439 & $29,625.10$ & $1,221.94$ & 0.481 & $(0.430-0.538)$ & $<0.0001$ & 0.478 & $(0.427-0.534)$ & $<0.0001$ \\
\hline$>56.0$ & 240 & 6,627 & $36,826.55$ & 651.70 & 0.245 & $(0.214-0.280)$ & $<0.0001$ & 0.256 & $(0.224-0.293)$ & $<0.0001$ \\
\hline \multicolumn{11}{|c|}{ Tertiles of cumulative dose of metformin therapy (grams) } \\
\hline Never users & 2,022 & 19,498 & $80,068.10$ & $2,525.35$ & 1.000 & & & 1.000 & & \\
\hline$<736$ & 480 & 6,434 & $20,985.04$ & $2,287.34$ & 0.975 & $(0.879-1.083)$ & 0.6395 & 0.893 & $(0.808-0.987)$ & 0.0260 \\
\hline $736-1918$ & 367 & 6,433 & $29,821.41$ & $1,230.66$ & 0.483 & $(0.432-0.540)$ & $<0.0001$ & 0.482 & $(0.431-0.539)$ & $<0.0001$ \\
\hline$>1918$ & 242 & 6,631 & $36,390.26$ & 665.01 & 0.247 & $(0.216-0.283)$ & $<0.0001$ & 0.262 & $(0.229-0.299)$ & $<0.0001$ \\
\hline \multicolumn{11}{|c|}{ Tertiles of units of defined daily dose of metformin therapy per day } \\
\hline Never users & 2,022 & 19,498 & $80,068.10$ & $2,525.35$ & 1.000 & & & 1.000 & & \\
\hline$<0.49$ & 387 & 6,434 & $26,985.17$ & $1,434.12$ & 0.574 & $(0.514-0.642)$ & $<0.0001$ & 0.564 & $(0.506-0.629)$ & $<0.0001$ \\
\hline $0.49-0.64$ & 351 & 6,434 & $28,633.36$ & $1,225.84$ & 0.476 & $(0.425-0.533)$ & $<0.0001$ & 0.483 & $(0.431-0.541)$ & $<0.0001$ \\
\hline$>0.64$ & 351 & 6,630 & $31,578.18$ & $1,111.53$ & 0.427 & $(0.381-0.479)$ & $<0.0001$ & 0.439 & $(0.392-0.492)$ & $<0.0001$ \\
\hline
\end{tabular}

Hemorrhoid was based on a diagnosis made at the out-patient clinics or during hospitalization.

a Unit of defined daily dose of metformin $=2$ grams.

IPTW: inverse probability of treatment weighting, HR: hazard ratio, Cl: confidence interval.

aspirin (models VI and VII) or the use of calcium channel blockers (models VIII and IX).

\section{DISCUSSION}

The is the first population-based observational study that showed an overall risk reduction of hemorrhoid associated with metformin use in patients with type 2 diabetes mellitus (Tables 2 and 3, Figures 2-5). A dose-response pattern could be seen in all analyses (Table 2, Figures 3-5).

The mechanisms of a reduced risk of hemorrhoid associated with metformin use require further investigation, but some basic research may provide tentative and reasonable explanations. Results from in vitro and in vivo studies suggested that metformin may exert cardiac and vascular protective effects via 5 -adenosine monophosphate-activated protein kinase (AMPK)-dependent and AMPK-independent pathways (Nesti and Natali, 2017). Pro-inflammation is a characteristic of insulin resistance (Grandl and Wolfrum, 2018). Metformin increases the expression of insulin receptor and activates tyrosine kinase, and therefore improves insulin resistance (Viollet et al., 2012). Additionally, by changing the composition of the gut microbiota, metformin use is associated with an increase in Akkermansia species, which have been shown to improve insulin resistance and reduce tissue inflammation (Hur and Lee, 2015). Metformin may also inhibit the transforming growth factor-beta one signaling pathways (Song et al., 2017), which are activated in cancer cells and several other human diseases involving autoimmunity, fibrosis and cardiovascular system (Serralheiro et al., 2017). Irritable bowel syndrome may cause hemorrhoid (Johannsson et al., 2005; Helvaci et al., 2009) 
TABLE 3 | Sensitivity analyses for estimating hazard ratios for hemorrhoid by metformin exposure.

\begin{tabular}{|c|c|c|c|c|c|c|c|c|c|c|}
\hline \multirow{2}{*}{$\begin{array}{l}\text { Model/Metformin } \\
\text { use }\end{array}$} & \multirow{2}{*}{$\begin{array}{c}\text { Incident } \\
\text { Case } \\
\text { number }\end{array}$} & \multirow{2}{*}{$\begin{array}{l}\text { Cases } \\
\text { Followed }\end{array}$} & \multirow[t]{2}{*}{ Person-years } & \multirow{2}{*}{$\begin{array}{c}\text { Incidence } \\
\text { rate } \\
\text { (per } \\
100,000 \\
\text { person-years) }\end{array}$} & \multicolumn{3}{|c|}{ Traditional Cox model } & \multicolumn{3}{|c|}{ IPTW model } \\
\hline & & & & & HR & $95 \% \mathrm{Cl}$ & p Value & HR & $95 \% \mathrm{Cl}$ & p Value \\
\hline
\end{tabular}

Unmatched cohort

I. Excluding two consecutive prescriptions of metformin spanning more than 4 months

\begin{tabular}{|c|c|c|c|c|c|c|c|c|c|c|}
\hline Never users & 2,025 & 19,523 & $80,153.12$ & $2,526.41$ & 1.000 & & & 1.000 & & \\
\hline Ever users & 2,515 & 52,075 & $220,925.10$ & $1,138.39$ & 0.437 & $(0.410-0.466)$ & $<0.0001$ & 0.448 & $(0.423-0.476)$ & $<0.0001$ \\
\hline \multicolumn{11}{|c|}{ I. Excluding patients treated with incretin-based therapies during follow-up } \\
\hline Never users & 2,002 & 18,510 & $75,573.20$ & $2,649.09$ & 1.000 & & & 1.000 & & \\
\hline Ever users & 7,588 & 119,286 & $526,011.63$ & $1,442.55$ & 0.533 & $(0.506-0.561)$ & $<0.0001$ & 0.540 & $(0.514-0.567)$ & $<0.0001$ \\
\hline \multicolumn{11}{|c|}{ II. Patients enrolled during 1999-2002 } \\
\hline Never users & 855 & 8,637 & $34,586.82$ & $2,472.04$ & 1.000 & & & 1.000 & & \\
\hline Ever users & 5,037 & 90,236 & $421,633.37$ & $1,194.64$ & 0.475 & $(0.440-0.513)$ & $<0.0001$ & 0.473 & $(0.440-0.508)$ & $<0.0001$ \\
\hline \multicolumn{11}{|c|}{ V. Patients enrolled during 2003-2005 } \\
\hline Never users & 1,170 & 10,886 & $45,566.30$ & $2,567.69$ & 1.000 & & & 1.000 & & \\
\hline Ever users & 3,174 & 62,111 & $270,852.70$ & $1,171.85$ & 0.440 & $(0.409-0.474)$ & $<0.0001$ & 0.455 & $(0.426-0.487)$ & $<0.0001$ \\
\hline \multicolumn{11}{|c|}{ V. Defining hemorrhoid as a primary diagnosis at hospitalization } \\
\hline Never users & 349 & 21,256 & $90,841.19$ & 384.19 & 1.000 & & & 1.000 & & \\
\hline Ever users & 1,381 & 169,495 & $783,364.48$ & 176.29 & 0.473 & $(0.417-0.536)$ & $<0.0001$ & 0.455 & $(0.405-0.512)$ & $<0.0001$ \\
\hline \multicolumn{11}{|c|}{ VI. Patients receiving aspirin } \\
\hline Never users & 1,211 & 11,985 & $48,552.79$ & $2,494.19$ & 1.000 & & & 1.000 & & \\
\hline Ever users & 5,211 & 96,528 & $442,205.39$ & $1,178.41$ & 0.457 & $(0.428-0.488)$ & $<0.0001$ & 0.464 & $(0.436-0.494)$ & $<0.0001$ \\
\hline \multicolumn{11}{|c|}{ VII. Patients not receiving aspirin } \\
\hline Never users & 814 & 7,538 & $31,600.33$ & $2,575.92$ & 1.000 & & & 1.000 & & \\
\hline Ever users & 3,000 & 55,819 & $250,280.68$ & $1,198.65$ & 0.477 & $(0.439-0.518)$ & $<0.0001$ & 0.462 & $(0.428-0.499)$ & $<0.0001$ \\
\hline \multicolumn{11}{|c|}{ VIII. Patients receiving calcium channel blockers } \\
\hline Never users & 1,315 & 13,118 & $53,210.21$ & $2,471.33$ & 1.000 & & & 1.000 & & \\
\hline Ever users & 5,168 & 94,952 & $432,049.30$ & $1,196.16$ & 0.469 & $(0.440-0.501)$ & $<0.0001$ & 0.477 & $(0.449-0.507)$ & $<0.0001$ \\
\hline \multicolumn{11}{|c|}{ X. Patients not receiving calcium channel blockers } \\
\hline Never users & 710 & 6,405 & $26,942.91$ & $2,635.20$ & 1.000 & & & 1.000 & & \\
\hline \multirow{2}{*}{\multicolumn{11}{|c|}{$\begin{array}{l}\text { Matched cohort } \\
\text { Excluding two }\end{array}$}} \\
\hline & & & & & & & & & & \\
\hline Never users & 2,022 & 19,498 & $80,068.10$ & $2,525.35$ & 1.000 & & & 1.000 & & \\
\hline Ever users & 349 & 7,170 & $30,027.67$ & $1,162.26$ & 0.456 & $(0.407-0.511)$ & $<0.0001$ & 0.459 & $(0.410-0.514)$ & $<0.0001$ \\
\hline \multicolumn{11}{|c|}{ I. Excluding patients treated with incretin-based therapies during follow-up } \\
\hline Never users & 1,999 & 18,486 & $75,489.58$ & $2,648.05$ & 1.000 & & & 1.000 & & \\
\hline Ever users & 1,034 & 15,806 & $68,477.61$ & $1,509.98$ & 0.567 & $(0.525-0.611)$ & $<0.0001$ & 0.567 & $(0.526-0.612)$ & $<0.0001$ \\
\hline \multicolumn{11}{|c|}{ II. Patients enrolled during 1999-2002 } \\
\hline Never users & 854 & 8,627 & $34,557.82$ & $2,471.22$ & 1.000 & & & 1.000 & & \\
\hline Ever users & 676 & 11,583 & $53,212.25$ & $1,270.38$ & 0.496 & $(0.448-0.550)$ & $<0.0001$ & 0.509 & $(0.460-0.563)$ & $<0.0001$ \\
\hline \multicolumn{11}{|c|}{ V. Patients enrolled during 2003-2005 } \\
\hline Never users & 1,168 & 10,871 & $45,510.28$ & $2,566.45$ & 1.000 & & & 1.000 & & \\
\hline Ever users & 413 & 7,915 & $33,984.45$ & $1,215.26$ & 0.464 & $(0.414-0.519)$ & $<0.0001$ & 0.471 & $(0.421-0.527)$ & $<0.0001$ \\
\hline \multicolumn{11}{|c|}{ V. Defining hemorrhoid as a primary diagnosis at hospitalization } \\
\hline Never users & 347 & 21,199 & $90,667.01$ & 382.72 & 1.000 & & & 1.000 & & \\
\hline Ever users & 179 & 21,199 & $96,460.75$ & 185.57 & 0.481 & $(0.401-0.577)$ & $<0.0001$ & 0.484 & $(0.404-0.579)$ & $<0.0001$ \\
\hline \multicolumn{11}{|c|}{ VI. Patients receiving aspirin } \\
\hline Never users & 1,208 & 11,966 & $48,487.73$ & $2,491.35$ & 1.000 & & & 1.000 & & \\
\hline Ever users & 647 & 12,019 & $53,843.20$ & $1,201.64$ & 0.473 & $(0.429-0.520)$ & $<0.0001$ & 0.479 & $(0.436-0.527)$ & $<0.0001$ \\
\hline \multicolumn{11}{|c|}{ VII. Patients not receiving aspirin } \\
\hline Never users & 814 & 7,532 & $31,580.38$ & $2,577.55$ & 1.000 & & & 1.000 & & \\
\hline Ever users & 442 & 7,479 & $33,353.50$ & $1,325.20$ & 0.510 & $(0.454-0.573)$ & $<0.0001$ & 0.512 & $(0.456-0.574)$ & $<0.0001$ \\
\hline \multicolumn{11}{|c|}{ VIII. Patients receiving calcium channel blockers } \\
\hline Never users & 1,312 & 13,099 & $53,141.72$ & $2,468.87$ & 1.000 & & & 1.000 & & \\
\hline Ever users & 721 & 13,036 & $58,168.46$ & $1,239.50$ & 0.493 & $(0.450-0.540)$ & $<0.0001$ & 0.500 & $(0.456-0.547)$ & $<0.0001$ \\
\hline \multicolumn{11}{|c|}{ X. Patients not receiving calcium channel blockers } \\
\hline Never users & 710 & 6,399 & $26,926.38$ & $2,636.82$ & 1.000 & & & 1.000 & & \\
\hline Ever users & 368 & 6,462 & $29,028.24$ & $1,267.73$ & 0.481 & $(0.423-0.546)$ & $<0.0001$ & 0.478 & $(0.422-0.542)$ & $<0.0001$ \\
\hline
\end{tabular}


and peptide YY plays an important role in the pathophysiology of irritable bowel syndrome (El-Salhy et al., 2020). Metformin has profound effects on gut hormone signaling including glucagonlike peptide 1 and peptide YY (Glossmann and Lutz, 2019). Whether metformin may prevent the development of hemorrhoid through its actions on gut hormones is an interesting research topic awaiting more in-depth investigation. Metformin may cause increased levels of growth differentiation factor 15; and this increase mediates its effect of body weight loss (Coll et al., 2020). Therefore, metformin may also protect against hemorrhoid by weight reduction following its use. Taken together, metformin may reduce the risk of hemorrhoid via multiple mechanisms by improving insulin resistance, reducing inflammation and fibrosis, affecting gut hormone signaling and weight reduction.

The findings of the present study extended the beneficial effects beyond glycemic control of metformin to the prevention of a very common clinical disease of hemorrhoid. There are some clinical implications. First, metformin has many beneficial effects beyond its glucose lowering effect. These include insulin sensitization, anti-inflammation, cardiovascular protection, anti-aging, anti-cancer and even anti-microbial effects (Maniar et al., 2017; Tseng, 2018a; Tseng, 2018b; Malik et al., 2018). Together with our recent studies, metformin may also exert protection against the development of venous diseases like varicose veins (Tseng, 2020) and hemorrhoid (findings of the present study). These provide good rationales for the use of metformin as the first-line therapeutic drug in the treatment of type 2 diabetes mellitus as recommended by major treatment guidelines (American Diabetes Association, 2017; Salvatore et al., 2020). Second, because all metformin-treated patients seemed to benefit from such a protective effect disregarding the units of DDD taken per day and the protective effect was mainly observed after a cumulative duration of 2 years or a cumulative dose of 750 grams, the use of metformin should be maintained to reach these thresholds when other antidiabetic drugs are added for better glycemic control. Third, the saving of the total healthcare expenditures for the management of other clinical diseases that can be prevented by the continuous use of metformin in the diabetes patients is expected to surpass the drug cost of metformin, an inexpensive drug that does not cause hypoglycemia by itself. Fourth, this observational study gives good rationale for initiating clinical trials to investigate the preventive and therapeutic effects of metformin on hemorrhoid, in either the diabetes patients or the non-diabetes people. However, at this moment, it is still not realistic or justified to recommend metformin for the prevention of hemorrhoid and hemorrhoid-associated complications until the findings are further confirmed by additional observation studies or by clinical trials. If such a protective effect can be confirmed, the clinical usefulness of metformin will be expanded.

In recent years, administrative databases have been popularly used to evaluate long-term safety or beneficial or side effects of medications in pharmacoepidemiological studies. These big data analyses are especially useful for outcomes with low incidence or when randomized trials are not practical. However, some methodological limitations should be carefully addressed.
These may include selection bias, prevalent user bias, immortal time bias and confounding by indication.

The present study was designed and conducted to address these potential methodological limitations. First, selection bias can be avoided by using the nationwide NHI database that covers $>99 \%$ of the population. Second, the potential risk of prevalent user bias was prevented by enrolling patients with new-onset diabetes mellitus and new users of metformin. Additionally, the exclusion of ever users of metformin who had ever been treated with other antidiabetic drugs before metformin was initiated (Figure 1) might have reduced the impacts of other antidiabetic drugs that could occur and be carried over to the period when metformin was initiated.

Third, we tried our best to reduce the immortal time bias. Immortal time refers to the follow-up period during which the outcome cannot happen, and immortal time bias can be introduced when treatment status or follow-up time is inappropriately assigned (Lévesque et al., 2010). We tried to exclude patients with uncertain diagnosis of diabetes mellitus by enrolling only patients who had been prescribed antidiabetic drugs for 2 or more times (Figure 1). Misclassification of treatment status with metformin was not likely because all prescription information was available in the NHI reimbursement database during the long follow-up period. The immortal time between the diagnosis of diabetes mellitus and the initiation of antidiabetic drugs and the immortal time during the short follow-up period of $<180$ days had been purposely excluded in the calculation of person-years. It is worthy to note that the immortal time pointed out by Lévesque et al. (2010) during the waiting period between drug prescription and dispense at hospital discharge would not happen in Taiwan because all patients can get their discharge drugs from the hospital at the time they are discharged.

Fourth, we aimed at reducing the confounding by indication by using the PS-matched cohort and the Cox regression incorporated with IPTW (Tables 2 and 3). Because all values of standardized difference were $<10 \%$ in the matched cohort (Table 1), the possibility of residual confounding from the covariates was small in the models created from the matched cohort. Additionally, the consistency of the findings in the unmatched cohort, in the analyses by using the traditional Cox regression and in the sensitivity analyses all supported that the results are robust and not liable to changes in different cohorts or by using different statistical methods.

Study limitations may include a lack of measurement data of confounders like biochemical and humoral profiles, anthropometric factors, lifestyle, physical activity, history of constipation and diarrhea, history of sexual intercourse, daily standing time, numbers of births and pelvic disease in women, cigarette smoking, alcohol drinking, dietary pattern, family history and genetic parameters. Visceral neuropathy or pudendal neuralgia may cause chronic constipation and hemorrhoid (Bharucha et al., 2013). However, we do not have such information in the database for analyses. Because the diagnosis of hemorrhoid was based on ICD-9-CM code without supportive laboratory data, it is possible that misclassification of hemorrhoid could not be entirely excluded. 
However, the hazard ratios would only be underestimated if the misclassifications were not differential in ever users and never users of metformin (Kesmodel, 2018). To further confirm the preventive role of metformin on hemorrhoid, sensitivity analyses were conducted by re-defining the outcome with a more stringent criterion of a primary diagnosis of hemorrhoid during hospitalization (Models V, Table 3). The estimated hazard ratios were very similar to those derived from the main analyses in Table 2. Finally, there is a possibility that some patients might not report their symptoms or signs related to hemorrhoid to their attending doctors and might have bought medications to treat their hemorrhoids by themselves. Because the coverage rate of NHI is very high and the patients always do not need to give extra payment if they get their medications for hemorrhoid at the same time when they receive their antidiabetic prescriptions, it is believed that the diabetes patients would rather report related symptoms to their doctors and requested medications for their hemorrhoids than buy over-the-counter medications by themselves that would cost extra expenses out of their pockets. Furthermore, if such misclassification was not differential between ever users and never users of metformin, the estimated hazard ratios would only be biased toward the null (Kesmodel, 2018).

There are some additional strengths. First, self-reporting bias could be much reduced by using medical records. Second, although detection bias related to different socioeconomic status can be a problem in some countries, this was less likely a problem here because the drug cost-sharing in the Taiwan's NHI healthcare system is low. Furthermore, much expense can be waived in veterans and in patients with low-income or when the patients receive prescription refills for chronic disease.

\section{CONCLUSION}

The findings of this study support a lower risk of hemorrhoid associated with chronic therapy of metformin in patients with type 2 diabetes mellitus in Taiwan when the cumulative duration is $>2$ years or the cumulative dose is $>750$ grams. However, confirmation in other populations is necessary. Because metformin does not cause hypoglycemia when used as a monotherapy and it is inexpensive and safe for long-term use, its protective effect on hemorrhoid is worthy of more

\section{REFERENCES}

American Diabetes Association (2017). Pharmacologic approaches to glycemic treatment. Diabetes Care 40 (Suppl. 1), S64-S74. doi:10.2337/dc17-S011

Austin, P. C., and Stuart, E. A. (2015). Moving towards best practice when using inverse probability of treatment weighting (IPTW) using the propensity score to estimate causal treatment effects in observational studies. Stat. Med. 34 (28), 3661-3679. doi:10.1002/sim.6607

Austin, P. C. (2013). The performance of different propensity score methods for estimating marginal hazard ratios. Stat. Med. 32 (16), 2837-2849. doi:10.1002/ $\operatorname{sim} .5705$ investigation, not only in patients with diabetes mellitus but also in non-diabetes people.

\section{DATA AVAILABILITY STATEMENT}

The datasets for this article are not publicly available because public availability of the dataset is restricted by local regulations to protect privacy.

\section{ETHICS STATEMENT}

The studies involving human participants were reviewed and approved by the Research Ethics Committee of the National Health Research Institutes. Written informed consent from the participants was not required in this study in accordance with the national legislation and the institutional requirements.

\section{AUTHOR CONTRIBUTIONS}

The author confirms being the sole contributor of this work and has approved it for publication.

\section{FUNDING}

The study was supported by the Ministry of Science and Technology (MOST 107-2221-E-002-129-MY3) of Taiwan and the Yee Fong Charity Foundation. The funders had no role in study design, data collection and analysis, decision to publish, or preparation of the manuscript.

\section{ACKNOWLEDGMENTS}

The study is based in part on data from the National Health Insurance Research Database provided by the Bureau of National Health Insurance, Department of Health and managed by National Health Research Institutes. The interpretation and conclusions contained herein do not represent those of Bureau of National Health Insurance, Department of Health or National Health Research Institutes.

Bharucha, A. E., Pemberton, J. H., and Locke, G. R., 3rd (2013). American Gastroenterological Association technical review on constipation. Gastroenterology 144 (1), 218-238. doi:10.1053/j.gastro.2012.10.028

Chang, Y. T., Tsai, H. L., Kung, Y. T., Yeh, Y. S., Huang, C. W., Ma, C. J., et al. (2018). Dose-dependent relationship between metformin and colorectal cancer occurrence among patients with type 2 diabetes-a nationwide cohort study. Transl. Oncol. 11 (2), 535-541. doi:10.1016/j.tranon.2018.02.012

Coll, A. P., Chen, M., Taskar, P., Rimmington, D., Patel, S., Tadross, J., et al. (2020). GDF15 mediates the effects of metformin on body weight and energy balance. Nature 578 (7795), 444-448. doi:10.1038/s41586-019-1911-y

Davis, B. R., Lee-Kong, S. A., Migaly, J., Feingold, D. L., and Steele, S. R. (2018). The American Society of Colon and Rectal Surgeons clinical practice guidelines for 
the management of hemorrhoids. Dis. Colon Rectum 61 (3), 284-292. doi:10. 1097/DCR.0000000000001030

Ekici, U., Kartal, A., and Ferhatoglu, M. F. (2019). Association between hemorrhoids and lower extremity chronic venous insufficiency. Cureus 11 (4), e4502. doi:10.7759/cureus.4502

El-Salhy, M., Hatlebakk, J. G., and Hausken, T. (2020). Possible role of peptide YY (PYY) in the pathophysiology of irritable bowel syndrome (IBS). Neuropeptides 79, 101973. doi:10.1016/j.npep.2019.101973

Glossmann, H. H., and Lutz, O. (2019). Pharmacology of metformin - an update. Eur. J. Pharmacol. 865, 172782. doi:10.1016/j.ejphar.2019.172782

Grandl, G., and Wolfrum, C. (2018). Hemostasis, endothelial stress, inflammation, and the metabolic syndrome. Semin. Immunopathol. 40 (2), 215-224. doi:10. 1007/s00281-017-0666-5

Helvaci, M. R., Algin, M. C., and Kaya, H. (2009). Irritable bowel syndrome and chronic gastritis, hemorrhoid, urolithiasis. Eurasian J. Med. 41 (3), $158-161$.

Hur, K. Y., and Lee, M. S. (2015). New mechanisms of metformin action: focusing on mitochondria and the gut. J. Diabetes Investig. 6 (6), 600-609. doi:10.1111/ jdi. 12328

Johannsson, H. O., Graf, W., and Påhlman, L. (2005). Bowel habits in hemorrhoid patients and normal subjects. Am. J. Gastroenterol. 100 (2), 401-406. doi:10. $1111 /$ j.1572-0241.2005.40195.x

Kesmodel, U. S. (2018). Information bias in epidemiological studies with a special focus on obstetrics and gynecology. Acta Obstet. Gynecol. Scand. 97 (4), 417-423. doi:10.1111/aogs.13330

Lévesque, L. E., Hanley, J. A., Kezouh, A., and Suissa, S. (2010). Problem of immortal time bias in cohort studies: example using statins for preventing progression of diabetes. BMJ 340, b5087. doi:10.1136/bmj.b5087

Lohsiriwat, V. (2012). Hemorrhoids: from basic pathophysiology to clinical management. World J. Gastroenterol. 18 (17), 2009-2017. doi:10.3748/wjg. v18.i17.2009

Malik, F., Mehdi, S. F., Ali, H., Patel, P., Basharat, A., Kumar, A., et al. (2018). Is metformin poised for a second career as an antimicrobial? Diabetes Metab. Res. Rev. 34 (4), e2975. doi:10.1002/dmrr.2975

Maniar, K., Moideen, A., Mittal, A., Patil, A., Chakrabarti, A., and Banerjee, D. (2017). A story of metformin-butyrate synergism to control various pathological conditions as a consequence of gut microbiome modification: genesis of a wonder drug?. Pharmacol. Res. 117, 103-128. doi:10.1016/j.phrs.2016.12.003

Mott, T., Latimer, K., and Edwards, C. (2018). Hemorrhoids: diagnosis and treatment options. Am. Fam. Physician 97 (3), 172-179. doi:10.1007/2F9783-319-53357-5_5

Nagaraj, S. V., Mori, A., and Reddy, M. (2019). Association of hemorrhoid vascular injuries with cigarette smoking-an evaluation with interesting prospects. Surg. J. (New York, N.Y.) 5 (4), e172-e176. doi:10.1055/s-0039-1700497

Nesti, L., and Natali, A. (2017). Metformin effects on the heart and the cardiovascular system: a review of experimental and clinical data. Nutr. Metabol. Cardiovasc. Dis. 27 (8), 657-669. doi:10.1016/j.numecd.2017.04.009

Parsons, L. S. (2020). Performing a 1:N case-control match on propensity score $\mathrm{http}: / / \mathrm{www} \cdot$ google.com.tw/url? $\mathrm{sa}=\mathrm{t} \& \mathrm{rct}=\mathrm{j} \& \mathrm{q}=\& \mathrm{esrc}=\mathrm{s} \&$ source=web\&cd=1\&ved= 0CBsQFjAAahUKEwibi7HllcnIAhUDoJQKHVeZA9A\&url=http\%3A\%2F\%2Fwww2. sas.com\%2Fproceedings\%2Fsugi29\%2F165-29.pdf\&usg=AFQjCNFOHGWYu8E8Bn4BolTUiJKtT987Q
Riss, S., Weiser, F. A., Schwameis, K., Riss, T., Mittlböck, M., Steiner, G., et al. (2012). The prevalence of hemorrhoids in adults. Int. J. Colorectal Dis. 27 (2), 215-220. doi:10.1007/s00384-011-1316-3

Sahebally, S. M., Ahmed, K., Cerneveciute, R., Iqbal, A., Walsh, S. R., and Joyce, M. R. (2017). Oral versus topical calcium channel blockers for chronic anal fissurea systematic review and meta-analysis of randomized controlled trials. Int. J. Surg. 44, 87-93. doi:10.1016/j.ijsu.2017.06.039

Salvatore, T., Pafundi, P. C., Morgillo, F., Di Liello, R., Galiero, R., Nevola, R., et al. (2020). Metformin: an old drug against old age and associated morbidities. Diabetes Res. Clin. Pract. 160, 108025. doi:10.1016/j.diabres.2020.108025

Serralheiro, P., Soares, A., Costa Almeida, C. M., and Verde, I. (2017). TGF- $\beta 1$ in vascular wall pathology: unraveling chronic venous insufficiency pathophysiology. Int. J. Mol. Sci. 18 (12), 2534. doi:10.3390/ijms18122534

Song, Y., Chen, Y., Li, Y., Lyu, X., Cui, J., Cheng, Y., et al. (2017). Metformin inhibits TGF- $\beta 1$-induced epithelial-to-mesenchymal transition-like process and stem-like properties in GBM via AKT/mTOR/ZEB1 pathway. Oncotarget 9 (6), 7023-7035. doi:10.18632/oncotarget.23317

Sun, Z., and Migaly, J. (2016). Review of hemorrhoid disease: presentation and management. Clin. Colon Rectal Surg. 29 (1), 22-29. doi:10.1055/s-00351568144

Tseng, C. H. (2012). Diabetes, metformin use, and colon cancer: a populationbased cohort study in Taiwan. Eur. J. Endocrinol. 167 (3), 409-416. doi:10.1530/ EJE-12-0369

Tseng, C. H. (2018a). Metformin and Helicobacter pylori infection in type 2 diabetes patients. Diabetes Care 41 (4), e42-e43. doi:10.2337/dc17-2551

Tseng, C. H. (2018b). Metformin decreases risk of tuberculosis infection in type 2 diabetes patients. J. Clin. Med. 7 (9), 264. doi:10.3390/jcm7090264

Tseng, C. H. (2017). Metformin is associated with a lower risk of colorectal cancer in Taiwanese patients with type 2 diabetes: a retrospective cohort analysis. Diabetes Metab. 43 (5), 438-445. doi:10.1016/j.diabet.2017.03.004

Tseng, C. H. (2020). Metformin reduces risk of varicose veins in patients with type 2 diabetes. Diabetes Metab. Res. Rev. 36 (2), e3206. doi:10.1002/dmrr. 3206

Viollet, B., Guigas, B., Sanz Garcia, N., Leclerc, J., Foretz, M., and Andreelli, F. (2012). Cellular and molecular mechanisms of metformin: an overview. Clin. Sci. (London, England) 122 (6), 253-270. doi:10.1042/CS20110386

Wang, Y. W., He, S. J., Feng, X., Cheng, J., Luo, Y. T., Tian, L., et al. (2017). Metformin: a review of its potential indications. Drug Des. Dev. Ther. 11, 2421-2429. doi:10.2147/DDDT.S141675

Yetkin, E., and Ozturk, S. (2018). Dilating vascular diseases: pathophysiology and clinical aspects. Int. J. Vasc. Med. 2018, 9024278. doi:10.1155/2018/9024278

Conflict of Interest: The author declares that the research was conducted in the absence of any commercial or financial relationships that could be construed as a potential conflict of interest.

Copyright $\odot 2021$ Tseng. This is an open-access article distributed under the terms of the Creative Commons Attribution License (CC BY). The use, distribution or reproduction in other forums is permitted, provided the original author(s) and the copyright owner(s) are credited and that the original publication in this journal is cited, in accordance with accepted academic practice. No use, distribution or reproduction is permitted which does not comply with these terms. 